\title{
Author Correction: Physics for neuromorphic computing
}

\author{
Danijela Marković, Alice Mizrahi, Damien Querlioz (1) and Julie Grollier(i)
}

Correction to: Nature Reviews Physics https://doi.org/10.1038/s42254-020-0208-2, published online 28 July 2020.

The originally published article contained an error in Fig. 3. The schematic in part b included a pre-synaptic spike that occurred after the post-synaptic spike and was shown as having an increasing weight, and the pre-synaptic spike that preceded the post-synaptic spike was shown as having a decreasing weight. In addition, the blue astrocyte in part a was referred to as grey in the caption. The order of the spikes has been corrected in part $\mathbf{b}$ of the figure and the colour of the astrocyte has been corrected in the caption. The figure and caption have been updated in the PDF and HTML versions of the article.

https://doi.org/10.1038/s42254-021-00358-7 I Published online 21 July 2021

(C) Springer Nature Limited 2021 